\title{
GADD45B as a Prognostic and Predictive Biomarker in Stage II Colorectal Cancer
}

\author{
Zhixun Zhao ${ }^{1,+}(\mathbb{D})$, Yibo Gao ${ }^{2,+}(\mathbb{D})$, Xu Guan ${ }^{3}$, Zheng Liu ${ }^{3}$, Zheng Jiang ${ }^{3}$, Xiuyun Liu ${ }^{4}$, \\ Huixin Lin ${ }^{5}$, Ming Yang ${ }^{3}$, Chunxiang Li ${ }^{2}$, Runkun Yang ${ }^{1}$, Shuangmei Zou ${ }^{4, *}$ \\ and Xishan Wang 1,3,* \\ 1 Department of Colorectal Surgery, The Second Affiliated Hospital of Harbin Medical University, \\ Harbin 150086, China; xunnna@126.com (Z.Z.); gaoyibo@cicams.ac.cn (Y.G.); yrkun0728@126.com (R.Y.) \\ 2 Department of Thoracic Surgery, National Cancer Center/National Clinical Research Center for \\ Cancer/Cancer Hospital, Chinese Academy of Medical Sciences and Peking Union Medical College, \\ Beijing 100020, China; lichunxiang@cicams.ac.cn \\ 3 Department of Colorectal Surgery, National Cancer Center/National Clinical Research Center for \\ Cancer/Cancer Hospital, Chinese Academy of Medical Sciences and Peking Union Medical College, \\ Beijing 100020, China; drguanxu@163.com (X.G.); zheng.liu@yale.edu (Z.L.); zone-j@hotmail.com (Z.J.); \\ yangming_0517@163.com (M.Y.) \\ 4 Department of Pathology, National Cancer Center/National Clinical Research Center for Cancer/Cancer \\ Hospital, Chinese Academy of Medical Sciences and Peking Union Medical College, Beijing 100020, China; \\ 13366818618@126.com \\ 5 Geneis (Beijing) Co., Ltd., Beijing 100102, China; linhx2@geneis.cn \\ * Correspondence: smzou@hotmail.com (S.Z.); wxshan1208@126.com (X.W.) \\ + These authors contributed equally to this work.
}

Received: 30 May 2018; Accepted: 10 July 2018; Published: 19 July 2018

\begin{abstract}
GADD45B acts as a member of the growth arrest DNA damage-inducible gene family, which has demonstrated to play critical roles in DNA damage repair, cell growth, and apoptosis. This study aimed to explore the potential relationship between $G A D D 45 B$ expression and tumor progression and evaluate the clinical value of GADD $45 B$ in stage II colorectal cancer (CRC). The expression patterns and prognostic value of GADD45B in CRC were analyzed based on The Cancer Genomic Atlas (TCGA). GADD45B expression features of 306 patients with stage II CRC and 201 patients with liver metastasis of $C R C$ were investigated using immunochemical staining on tissue microarrays. Afterward, survival analysis and stratification analysis were performed in stage II to explore the prognostic and predictive significance of GADD45B. Overexpressed GADD45B is associated with poorer prognosis for CRC patients both in overall survival (OS) $(p<0.001)$ and disease-free survival (DFS) $(p=0.001)$ based on the TCGA database. Analysis results according to the stage II CRC cohort and the liver metastatic CRC cohort revealed that GADD45B was gradually upregulated in normal mucosa including primary colorectal cancer (PCC). Colorectal liver metastases (CLM) tissues were arranged in order (normal tissue vs. PCC $p=0.005$ and PCC vs. CLM $p=0.001$ ). The low GADD45B group had a significantly longer five-year OS $(p=0.001)$ and progression-free survival (PFS) $(p<0.001)$ than the high GADD45B group for the stage II patients. The multivariate Cox regression analysis results proved that the expression level of $G A D D 45 B$ was an independent prognostic factor for stage II after radical surgery (OS: Hazard Ratio (HR) 0.479, [95\% confidence interval (CI) 0.305-0.753] and PFS:HR 0.490, [95\% CI 0.336-0.714]). In high GADD45B expression subgroup of stage II cohort, the patients who underwent adjuvant chemotherapy had longer PFS than those who did not $(p=0.008)$. High expression levels of GADD45B is an independent prognostic factor of decreased OS and PFS in stage II CRC patients. The stage II CRC patients with high GADD45B expression might benefit from adjuvant chemotherapy.
\end{abstract}


Keywords: colorectal cancer; the growth arrest DNA damage-inducible 45 beta (GADD45B); prognosis; stage II; biomarkers; adjuvant chemotherapy

\section{Introduction}

Colorectal cancer (CRC) is one of the most common cancers worldwide. Colorectal cancer is threatening public health. In China, CRC new cases and specific death estimates were approximately 191,000 and 376,300 annually, respectively [1]. On account of the prevalence of early CRC screening, the health outcomes of patients diagnosed with stage I or stage II CRCs has improved. However, the treatment options of early stage CRC, especially stage II, remains controversial. About $20 \%$ of the CRC patients who underwent curative surgery alone will develop systemic metastasis [2]. In addition, the previous study revealed that chemotherapy could improve survival of stage II CRC patients but absolute improvement in survival was less than 5\% [3]. Furthermore, the adverse events of adjuvant chemotherapy have an impact on the quality of life of patients [4]. Therefore, it is essential to identify valuable biomarkers to stratify the tumor progression risk after surgery in stage II CRC. In this way, patients who were predicted to have metastasis might get a benefit from the chemotherapy. Conversely, low-risk patients could avoid the associated adverse effects and save themselves the cost of chemotherapy.

The GADD45 family, which consists of GADD45A (GADD45 $\alpha$, DDIT1), GADD45B (GADD45 $\beta$, Myd118), and GADD45G (GADD45 $\gamma$, cytokine-responsive 6, CR6), participates in many cellular processes associated with cell growth regulation and the stress signaling pathway [5]. The proteins encoded by the GADD45 gene family are small (18kDa), conservative, and have homology and high acidity [6]. GADD 45B shares the common functions of the GADD45 family, which is associated with DNA damage repair, cell growth, apoptosis, and anti-tumor immune responses [7,8]. Meanwhile, GADD45B might play critical roles in the tumorigenesis of human embryonic carcinoma [9], hepatocellular carcinoma [10], and pituitary gonadotrope tumors [11]. GADD45 $\beta$ is related to NF-kB, which is known to influence tumorigenesis, cancer cell survival, apoptosis, invasion, and metastasis and the GADD45 family are essential mediators of cell survival in cancer cells with implications for cancer chemotherapy and novel drug discovery [9]. A recent study implicated the carcinogenesis function and potential prognostic value of GADD45B for CRC [12]. However, the role of GADD45B expression in the prognostic value and chemotherapy-related predictive significance in stage II CRC remains uncertain, which should gain more attention.

In the present study, we first evaluated the expression patterns of GADD45B in CRC and assessed prognostic significance based on The Cancer Genomic Atlas (TCGA). We further investigated the GADD $45 B$ expression features of stage II and liver metastatic CRC using immunochemical staining according to the data in our cohort. Lastly, survival analysis and stratification analysis were performed in stage II to explore the prognostic and predictive value of GADD45B.

\section{Materials and Methods}

\subsection{Patients}

The specimens in this study were collected from the patients who underwent the surgical resection between 2006 and 2012 at the Cancer Institute \& Hospital, Chinese Academy of Medical Sciences, Peking Union Medical College. All the diagnoses were confirmed according to the 7th edition American Journal of Critical Care (AJCC) TNM Classification. The inclusive criteria of stage II include the following: (A) AJCC pathology staging was stage II (T3-4NOM0); (B) no systemic or chemotherapy before the surgery; $(\mathrm{C})$ the case can provide complete clinical information such as age, gender, tumor location, histology, differentiation, malignant tumors TNM classification, adjuvant therapy regime, follow-up information, and more. At the same time, we utilized the primary tumor and matched 
metastatic liver specimen to establish another simultaneous liver metastatic CRC (LMCRC) cohort. Totally, 306 cases of stage II CRC and 201 cases of simultaneous LMCRC were collected based on the inclusive criteria. All subjects gave their informed consent for inclusion before they participated in the study. The study was conducted in accordance with the Declaration of Helsinki, the Clinical Research Ethics Committee of Cancer Institute \& Hospital, Chinese Academy of Medical Sciences approved this study (NCC2016JZ-06). All the patients were followed up every three months until 31 December 2017.

\subsection{Genomic Analyses in the Public Dataset}

To investigate the expression pattern and prognostic value of $G A D D 45 B$, we analyzed colon cancer in 270 cases and rectal cancer in 92 cases provided by the TCGA project (Table S3). The Box Plots was generated to compare the GADD $45 B$ expression level between the tumor and normal tissues of CRC. The violin plots were created based on the patients' pathological stages and GADD45B expression features. The Kaplan-Meier curves were plotted according to the expression value of GADD45B. The gene expression profiling interactive analysis (GEPIA) (http://gepia.cancer-pku.cn/index.html) is used for batch TCGA data processing and visualization in this study [13].

\subsection{Tissue Microarray and Immunohistochemistry}

The stage II tissue microarrays (TMAs) consisted of the tumor sample and matched normal tissue from each patient and LMCRC TMAs consisted of the primary colorectal cancer (PCC), colorectal liver metastases (CLM), normal intestinal mucosa, and normal liver tissue from each patient. All tumor tissue microarrays were built after being verified by hematoxylin and eosin (H\&E) staining. The punched sample measured $1.0 \mathrm{~mm}$ and was obtained from the center of the tumor. All the tissue microarrays were assessed by using immunohistochemical (IHC) staining and an anti-GADD45B antibody (ab105060; 1:500; Abcam; Cambridge, UK). The IHC staining is referenced to immunohistochemistry protocols published by the Abcam Company online [14]. The expression of the TMAs was scored by two independent pathologists in a blind study, according to the Staining Index (SI) that was previously used to assess the expression pattern of GADD45B [15,16]. The SI score was calculated by multiplying the staining intensity $(0$, negative; 1 , weak; 2 , moderate; 3 , strong) and the percentage of positive stained cells (no staining, $0 ; 1-10 \%, 1 ; 11-50 \%, 2 ; 50-100 \%, 3$ ). In this study, a moderate/strong cytoplasm staining of $(\mathrm{SI}=3-9)$ was defined as positive staining while a weak or negative staining ( $\mathrm{SI}=0-2)$ was defined as negative staining. Representative staining of $G A D D 45 B$ in the specimens was illustrated in Figure 1. The positive rate refers to the proportion of GADD45B positive staining samples especially positive rate = positive samples / (positive samples + negative samples). 
A

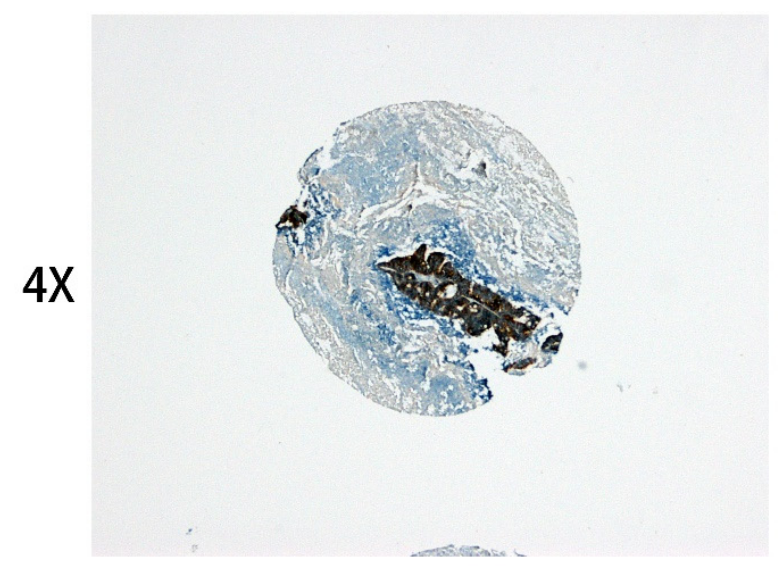

GADD45B high expression
B

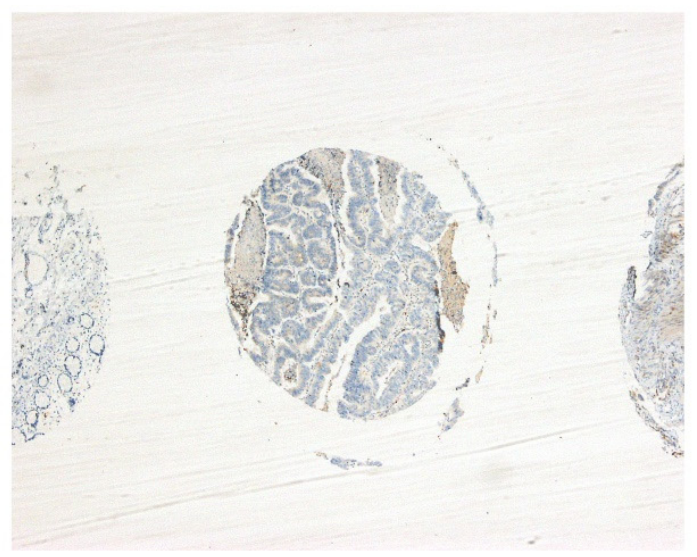

GADD45B low expression

Figure 1. Cont.

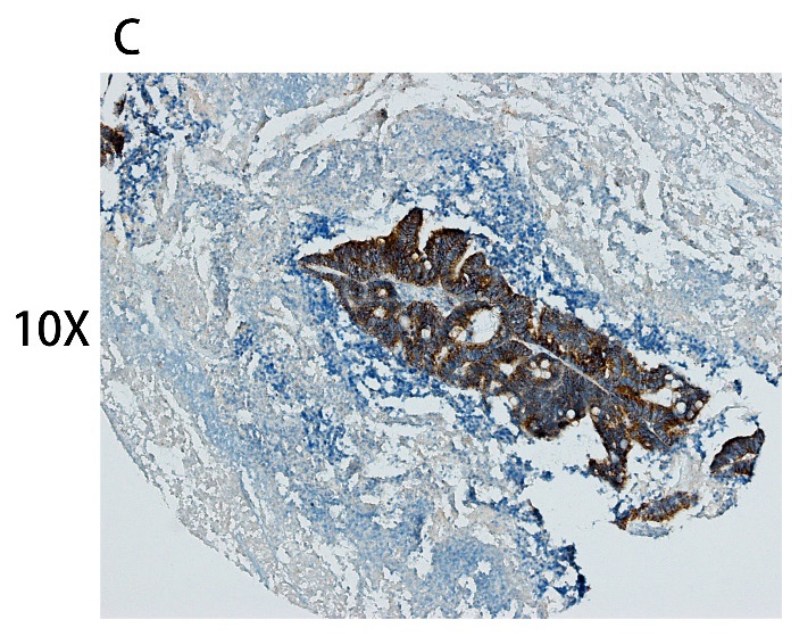

GADD45B high expression
D

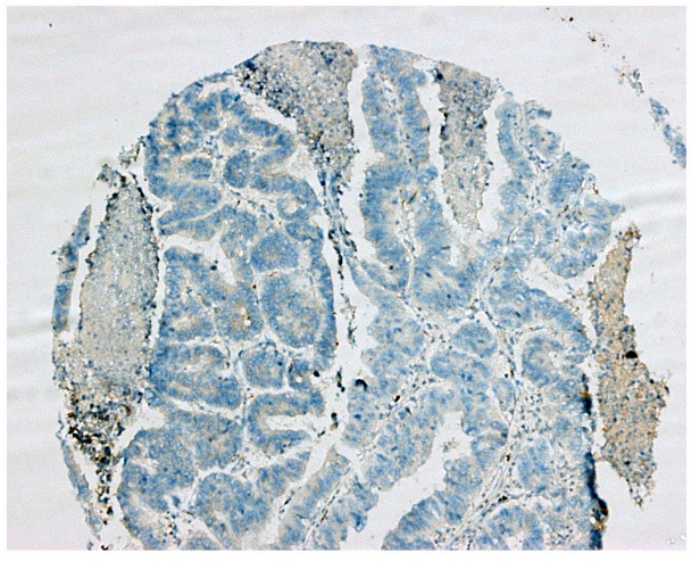

GADD45B low expression

Figure 1. Representative Immunohistochemistry staining pictures of $G A D D 45 B$ expression in colorectal cancer tissues. Tissue high expression $(4 \times$ for $(A), 10 \times$ for $(C))$ and low expression $(4 \times$ for $(B), 10 \times$ for $(\mathbf{D})$ ) for the $G A D D 45 B$ protein are shown. Each of the punched samples is $1.0 \mathrm{~mm}$ in length in the tissue microarrays.

\subsection{Statistical Analysis}

The student's $t$-test was used to compare the difference of the data between two groups. The Chi-square test or Fisher exact test was used to evaluate categorical data. Survival curves were plotted according to the Kaplan-Meier method and the log-rank test was used to compare the OS (overall survival), DFS (disease-free survival), and PFS (progression-free survival) in the study cohorts. Univariate and multivariate analysis for CRC prognosis were undertaken using the Cox proportional hazards regression model. The calculations were performed with International Business Machines (IBM) SPSS Statistics version 24.0 software program and $\mathrm{R}$ version 3.3.3. A value of $p<0.05$ was considered significant. 


\section{Results}

3.1. High GADD45B Expression Is Associated with Poorer Overall Survival and Disease-Free Survival in Colorectal Cancer Patients from the Public Database

We analyzed the GADD $45 B$ expression level of the CRC cases from the TCGA database and there was no significant difference between the PCC and normal tissues (NT) in both the colon cancer (COAD) and rectal cancer (READ) dataset (Figure 2A). Meanwhile, the average expression level of GADD45B turned out to have a rising trend as the development of the TNM pathology stage (Figure 2B). As shown in the Kaplan-Meier curves (Figure 2C,D), overexpressed GADD45B is associated with a poorer prognosis for CRC patients both in OS and DFS ( $p=0.014$ for OS; $p=0.041$ for DFS). 
A
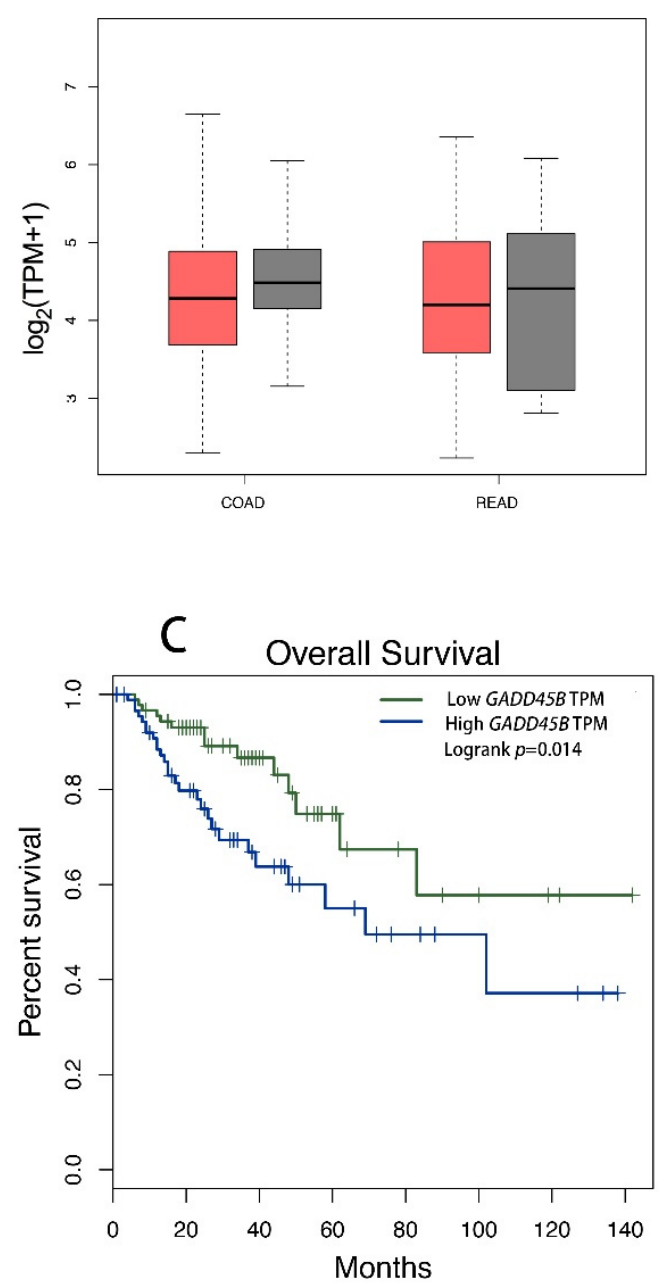

B
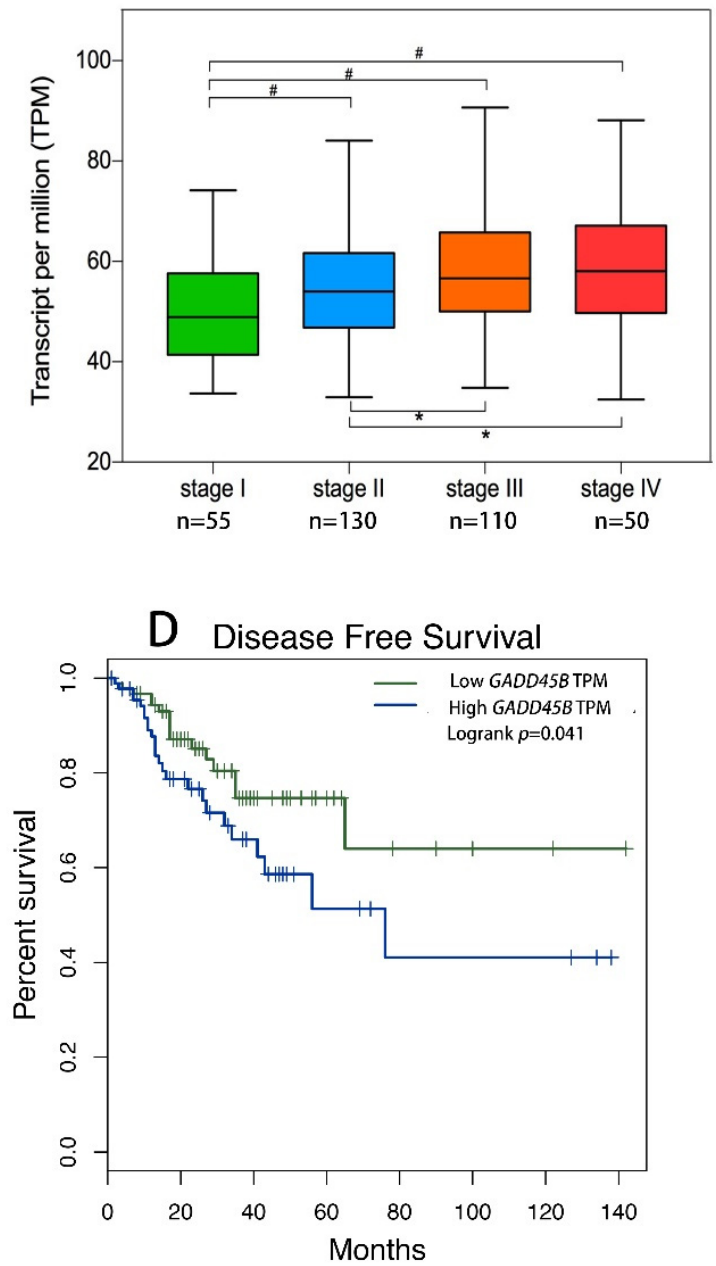

Figure 2. Expression of the GADD45B protein in CRC and Kaplan-Meier Curves of overall survival (OS) and disease-free survival (DFS) based on The Cancer Genomic Atlas (TCGA) database. The expression of $G A D D 45 B$ in colon cancer (COAD) and rectal cancer (READ) dataset was analyzed by gene expression profiling interactive analysis (GEPIA). The tumor was represented by a red color and the normal tissue was represented by a grey color (A). The GADD45 B expression box plots were generated based on CRC patient pathological major TNM staging (B). The most extreme value from bottom to top in the box plot represents the minimum value, the lower quartile, the median, the upper quartile, and the maximum value. The method for differential gene expression analysis is one-way Analysis of Variance (ANOVA) using the pathological stage as a variable for calculating differential expression. The GADD45B high expression group was associated with decreased OS (C) and DFS (D) in CRC according to the data from TCGA, which were calculated using a log-rank test. CRC: Colorectal cancer, TPM: Transcript per million, \# represents $p$-value $<0.01$, and * represents $p$-value $<0.05$.

\subsection{The GADD45B Expression Pattern in Stage II Colorectal Cancer and Liver Metastatic Colorectal Cancer Cohort}

To validate the results analyzed by the public database, we detected the expression patterns of GADD $45 B$ in the stage II CRC cohort and the LMCRC cohort. In these two cohorts, 306 cases of stage II CRC and 201 cases of simultaneous LMCRC included respectively. All the IHC staining results have been listed in Table 1 . In the stage II cohort, there was no significant difference regarding GADD $45 B$ protein expression in between the tumor tissue and the corresponding normal tissue (43.14\% vs. $36.93 \%, p=0.117$ ). Based on the follow-up data, stage II was divided into the non-progression group (195 cases) and the progression group (111 cases) and 31 cases liver metastasis included in 
the latter group. Immunohistochemistry staining indicated that the progression group had a higher GADD $45 B$ expression positive rate than the non-progression group $(50.45 \%$ vs. $38.97 \%, p=0.004)$.

In the LMCRC cohort, GADD45B protein expression was detected in 105/201 (52.24\%) of the PCC samples and 137/201 (68.16\%) of the CLM samples (Table 1). At the same time, 77 cases $(38.31 \%)$ of adjacent normal mucosa and 95 cases $(47.26 \%)$ of the normal liver tissue showed staining-positive. Therefore, at protein levels, GADD45B was gradually upregulated in normal mucosa, PCC, and CLM tissues in order (normal tissue vs. PCC $p=0.005$ and PCC vs. CLM $p=0.001$ ).

We further compared the GADD $45 B$ expression of the primary tumor between stage II and LMCRC, and the results demonstrated that the latter was significantly upregulated (43.14\% vs. $52.24 \%$, $p=0.045)$. We also analyzed the GADD $45 B$ expression pattern in the primary tumor between stage II with liver metastasis after surgery and the simultaneous liver metastatic group. No significant difference was found $(52.24 \%$ vs. $61.29 \%, p=0.347)$.

Table 1. The GADD45B expression pattern in different samples by immunohistochemical (IHC) staining.

\begin{tabular}{|c|c|c|c|c|}
\hline & Sample & GADD45B-Positive & GADD45B-Negative & Positive Rate \\
\hline \multirow{5}{*}{ Stage II } & Tumor & 132 & 174 & $43.14 \%$ \\
\hline & Normal Tissue & 113 & 193 & $36.93 \%$ \\
\hline & Progression & 60 & 51 & $50.45 \%$ \\
\hline & Non-progression & 72 & 123 & $38.97 \%$ \\
\hline & Recurrence of Liver Metastasis after Surgery & 19 & 12 & $61.29 \%$ \\
\hline \multirow{4}{*}{ LMCRC } & Primary Tumor & 105 & 96 & $52.24 \%$ \\
\hline & Normal Colorectal Mucosa & 77 & 124 & $38.31 \%$ \\
\hline & Liver Metastasis & 137 & 64 & $68.16 \%$ \\
\hline & Normal Liver Tissue & 95 & 106 & $47.26 \%$ \\
\hline
\end{tabular}

Stage II: Stage II colorectal cancer, LMCRC: Liver metastatic colorectal cancer, Progression: Tumour recurrence after surgery in 5 years, Non-Progression: No recurrence signs after surgery in 5 years.

\subsection{The High GADD45B Expression Is Associated with Shorter Overall Survival and Progression-Free Survival in Stage II Colorectal Cancer}

The association between the GADD45B expression of stage II primary and LMCRC patient pathologic characteristics is summarized in Table 2. However, the clinicopathologic information such as age, gender, tumor location, gross pathology type, differentiation grade, $\mathrm{T}$ stage, preoperative carcinoembryonic antigen (CEA) level, preoperative carbohydrate antigen 19-9(CA19-9) level, and microsatellite instability (MSI) status had no significant correlation with the GADD45B expression.

Table 2. Correlation between the intensity of GADD45B expression and the clinical profiles of stage II colorectal cancer.

\begin{tabular}{|c|c|c|c|c|}
\hline Factor & Total & GADD45B HIGH (\%) & GADD45B LOW (\%) & $p$ Value \\
\hline \multicolumn{5}{|l|}{ Age (years) } \\
\hline$<65$ & 216 & $89(41.2)$ & $127(58.8)$ & 0.290 \\
\hline$\geq 65$ & 90 & $43(47.8)$ & $47(52.2)$ & \\
\hline \multicolumn{5}{|l|}{ Gender } \\
\hline Male & 183 & $78(42.6)$ & $105(57.4)$ & 0.825 \\
\hline Female & 123 & $54(43.9)$ & $69(56.1)$ & \\
\hline \multicolumn{5}{|l|}{ Tumor Location } \\
\hline Colon & 143 & $59(72.4)$ & $84(27.6)$ & 0.836 \\
\hline Rectum & 163 & $73(71.3)$ & $90(28.7)$ & \\
\hline \multicolumn{5}{|l|}{ Gross Pathological Type } \\
\hline Prominence & 171 & $64(41.3)$ & $107(58.7)$ & 0.534 \\
\hline Ulceration \& Infiltration & 135 & $68(44.8)$ & $67(55.2)$ & \\
\hline \multicolumn{5}{|l|}{ Grade } \\
\hline High & 34 & $18(52.9)$ & $16(47.1)$ & 0.409 \\
\hline Middle & 240 & $102(42.5)$ & $138(57.5)$ & \\
\hline Low & 32 & $12(37.5)$ & $20(62.5)$ & \\
\hline
\end{tabular}


Table 2. Cont.

\begin{tabular}{|c|c|c|c|c|}
\hline Factor & Total & GADD45B HIGH (\%) & GADD45B LOW (\%) & $p$ Value \\
\hline \multicolumn{5}{|l|}{ T Stage } \\
\hline T3 & 286 & 125 (43.7) & $161(56.3)$ & \multirow[t]{2}{*}{0.175} \\
\hline $\mathrm{T} 4$ & 20 & $17(56.7)$ & $13(43.3)$ & \\
\hline \multicolumn{5}{|l|}{ Adjuvant Therapy } \\
\hline Chemotherapy & 131 & $53(40.5)$ & $78(59.5)$ & \multirow[t]{3}{*}{0.377} \\
\hline Radiotherapy & 63 & $32(50.8)$ & $31(49.2)$ & \\
\hline No & 112 & $47(42.0)$ & $65(58.0)$ & \\
\hline \multicolumn{5}{|c|}{ Preoperative CEA Level(ng/mL) } \\
\hline$\leq 5$ & 244 & 101 (41.4) & $143(58.6)$ & \multirow[t]{2}{*}{0.222} \\
\hline$>5$ & 62 & $31(50.0)$ & $31(50.0)$ & \\
\hline \multicolumn{5}{|c|}{ Preoperative CA19-9 Level(U/mL) } \\
\hline$\leq 37$ & 282 & $119(42.2)$ & $163(57.8)$ & \multirow[t]{2}{*}{0.182} \\
\hline$>37$ & 23 & $13(56.5)$ & $10(43.5)$ & \\
\hline \multicolumn{5}{|l|}{ MSI } \\
\hline MSS & 268 & $120(44.8)$ & $148(55.2)$ & \multirow[t]{2}{*}{0.124} \\
\hline MSI & 38 & $12(31.6)$ & $26(68.4)$ & \\
\hline
\end{tabular}

CEA: Carcinoembryonic antigen; CA19-9: Carbohydrate antigen 19-9; MSI: Microsatellite instability; MSS: Microsatellite stable; T stage: Tumour stage.

To identify the prognosis value of GADD45B expression in CRC, the Kaplan Meier survival analyses were conducted in stage II. The median follow-up was more than 59 months and 78 cases of death, and 111 relapsed patients included. Kaplan Meier analysis revealed that in stage II patients, the low GADD45B group had a significantly longer five-year OS and PFS than the high GADD45B group ( $p=0.001$ for OS and $p<0.001$ for PFS; Figure 3A,B).

A Stage II CRC

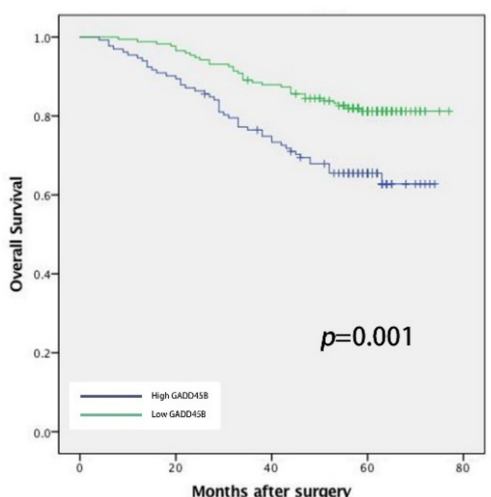

C Stage II CRC

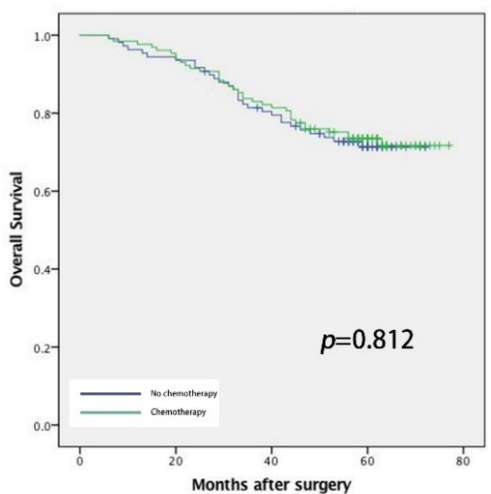

B Stage II CRC

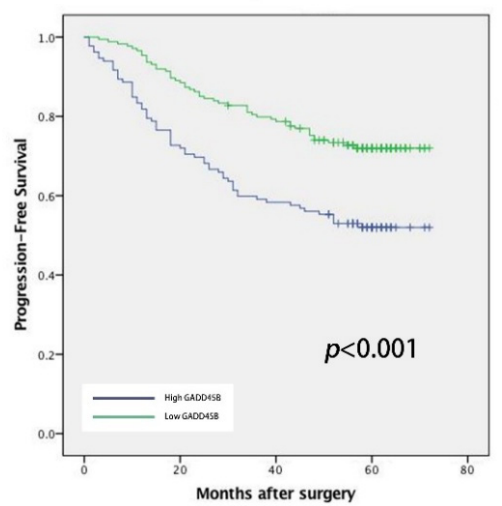

D Stage II CRC

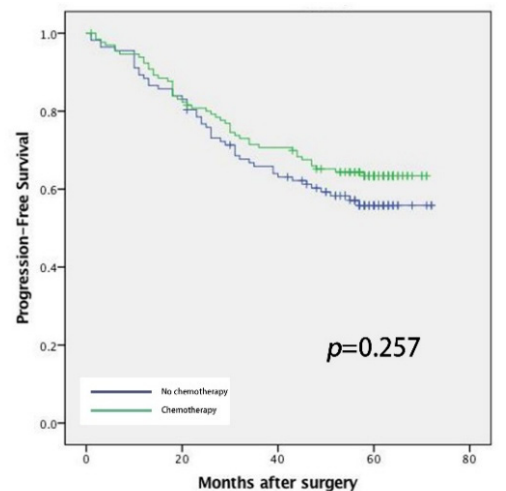

Figure 3. Cont. 

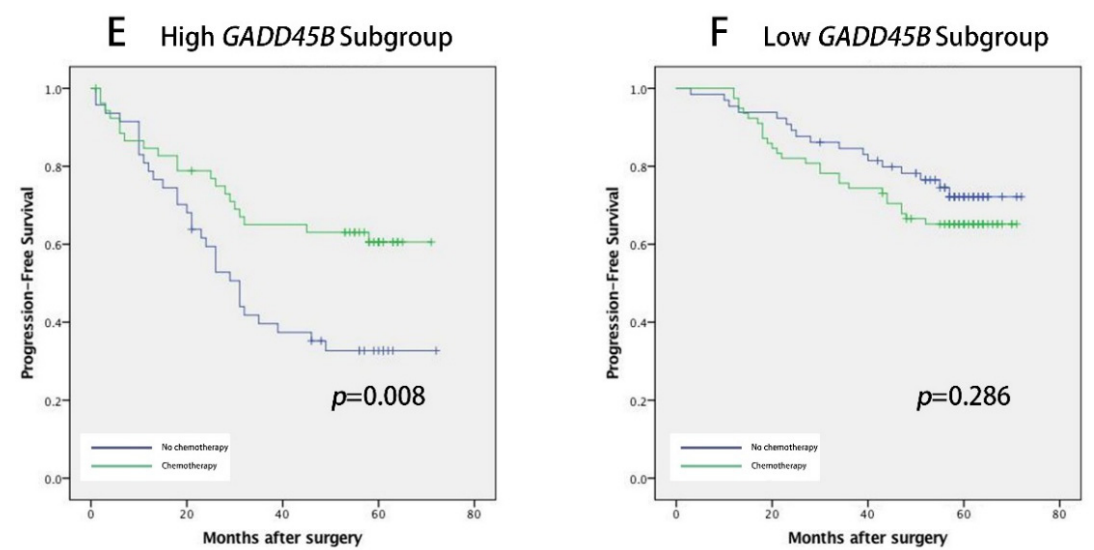

Figure 3. Prognostic power of GADD45B in stage II CRC cohort CRC cohort Kaplan-Meier analyses of overall survival and progression-free survival in patients with CRC based on the expression of GADD45B. Overall survival and progression-free survival (PFS) accord to GADD45B expression in stage II CRC (A,B). The relationship between GADD45B expression and PFS benefits from adjuvant chemotherapy in patients with stage II CRC. There was no significant difference in OS and PFS between the patients who received chemotherapy and the patients who never received the adjuvant therapy after surgery (C,D). Treatment with 5-Fluorouracil-based (5-FU-based) chemotherapy was associated with a higher rate of PFS in the GADD45B high group (E) but not in the GADD45B low group (F).

\subsection{GADD45B Expression Is an Independent Prognostic Factor in Stage II}

Univariate Cox regression analysis confirmed that high GADD45B was associated with a worse prognosis significance for OS (HR 0.459, [95\% CI 0.292-0.721]) and PFS (HR 0.480, [95\% CI 0.330-0.699], Tables 3 and 4, Tables S1 and S2). In the multivariate Cox regression analysis, high GADD45B expression was also significantly associated with the poorer rate of OS (HR 0.479, [95\% CI 0.305-0.753]) and PFS (HR 0.490, [95\% CI 0.336-0.714], Tables 3 and 4, Tables S1 and S2). The results proved that low-level expression of GADD $45 B$ was an independent prognostic factor for stage II after radical surgery. Furthermore, neurological involvement was also a significant independent prognostic factor for PFS in the stage II cohort.

Table 3. Cox analyses of potential prognostic factors for overall survival in the stage II CRC cohort.

\begin{tabular}{ccccccccc}
\hline \multirow{2}{*}{ Factor } & \multirow{2}{*}{ Comparison } & \multicolumn{3}{c}{ Univariate Analysis } & \multicolumn{3}{c}{ Multivariate Analysis } \\
\cline { 3 - 8 } & & HR & $\mathbf{9 5 \%}$ CI & $p$ Value & HR & $\mathbf{9 5 \% ~ C I ~}$ & $p$ Value \\
\hline MSI Status & MSI vs. MSS & 0.343 & $0.126-0.939$ & 0.037 & 0.379 & $0.138-1.039$ & 0.059 \\
GADD45B Expression & LOW vs. HIGH & 0.459 & $0.292-0.721$ & 0.001 & 0.479 & $0.305-0.753$ & 0.001 \\
\hline
\end{tabular}

HR: Hazard ratio; CI: confidence interval.

Table 4. Cox analyses of potential prognostic factors for progression-free survival in the stage II CRC cohort.

\begin{tabular}{|c|c|c|c|c|c|c|c|}
\hline \multirow{2}{*}{ Factor } & \multirow{2}{*}{ Comparison } & \multicolumn{3}{|c|}{ Univariate Analysis } & \multicolumn{3}{|c|}{ Multivariate Analysis } \\
\hline & & HR & $95 \%$ CI & $p$ Value & HR & $95 \% \mathrm{CI}$ & $p$ Value \\
\hline Neurological Involvement & Present vs. Absent & 2.222 & $1.191-4.145$ & 0.012 & 2.066 & $1.107-3.858$ & 0.023 \\
\hline GADD45B Expression & LOW vs. HIGH & 0.480 & $0.330-0.699$ & 0.0001 & 0.490 & $0.336-0.714$ & 0.0002 \\
\hline
\end{tabular}

\subsection{Expression of GADD45B Could Predict the Benefit from the Adjuvant Chemotherapy in Stage II Colorectal Cancer}

Next, we investigated the potential role of $G A D D 45 B$ as a predictor of effectiveness of chemotherapy in stage II. In this cohort, 131 patients received the 5-Fluorouracil-based (5-FU-based) adjuvant chemotherapy, 63 lower rectal cancer patients underwent radiotherapy (50 gray (Gy)), and 112 patients didn't take any adjuvant therapy after surgery. There appeared to be no significant 
difference in OS and PFS between the patients who received chemotherapy and the patients who never received the adjuvant treatment after surgery ( $p=0.812$ for OS and $p=0.257$ for PFS; Figure 3C,D). To assess the predictive value of GADD45B for an adjuvant chemotherapy benefit, we compared the PFS of stage II CRC patients who underwent adjuvant chemotherapy or not in the subgroup after being stratified by GADD45B expression. In the high GADD45B expression subgroup, chemotherapy was associated with a higher rate of PFS ( $p=0.008$; Figure 3E), but this relationship did not exist in the subgroup of low GADD $45 B$ expression ( $p=0.286$, Figure $3 F$ ). The test of interaction results indicated that the benefit associated with adjuvant chemotherapy in the high GADD45B expression group was superior to the low GADD45B expression group $(p=0.027)$.

\section{Discussion}

In the present study, we evaluated the GADD45B expression pattern of different $C R C$ development stages and prognostic values of GADD45B in stage II CRC. We first explored expression features of GADD45B in CRC based on the TCGA database and two CRC cohorts. As illustrated in the violin figure plotted by GEPIA, the expression level of GADD45B increased due to the development of the TNM stage. The results analyzed by our cohorts also indicated that the positive rate of GADD45B expression was rising in the primary tumor of stage II, PCC, and CLM of liver metastasis in order. Moreover, the IHC positive rate of GADD $45 B$ of the stage II progression group was higher than the non-progression group, but it turned out to have no significant difference in the primary tumor between stage II with liver metastasis after surgery and the simultaneous liver metastatic group. An associated study indicated that $G A D D 45 B$ contributed to tumor progression rather than the initiation in hepatocellular carcinoma and ovarian cancer [17]. Therefore, we infer that GADD45B may also be regarded as a potential tumor progression predictive marker in CRC.

When we made a survival analysis based on the TCGA data, the results suggested that the expression of GADD $45 B$ was significantly associated with the prognosis of CRC both in OS and PFS. Subsequently, the survival analysis was performed in the stage II cohort to validate the prognostic significance in this stage. It was shown that high expression of GADD45B was an independent prognostic factor of decreased OS and PFS accounting for stage II CRC. It was found that GADD45B had a predictive value to estimate the benefit from 5-FU-based chemotherapy in stage II. Additionally, in the previous studies, GADD45B was demonstrated to induce apoptosis and repair DNA damage in CRC cell lines, which plays a role in the tumor suppressor gene. This result seemed to be inconsistent with our study. However, a study published by Wang et al. [12] implicated that GADD45B might lose its normal functions and promote carcinogenesis and tumor progression in CRC tissues. At the same time, with the accumulation of DNA damage in tumor progression, the GADD45B expression level will improve feedback.

GADD45Beta is a stress-activated protein that plays a vital role in regulating apoptosis, proliferation, and DNA repair. It has been demonstrated to be an indicator for predicting clinical outcomes of gastric cancer [18], ovarian cancer [19], and glioma [20] according to the previous studies. Verzella et al. found that elevated GADD45B expression correlated with rapid disease progression in 13 of the top 15 solid cancers for mortality and the patient cohorts expressing high GADD45B levels exhibited significantly shorter recurrence-free survival and OS than the corresponding cohorts, which expressed low GADD45B messenger-RNA (mRNA) levels [16]. Even though a previous study has revealed the prognostic value of GADD45B in CRC, its validation cohort only included 152 cases with stage II-III [12]. Our study was performed to better understand the adjuvant chemotherapy indication-related problem for stage II patients with better clinical value. The results suggested that the benefit associated with adjuvant chemotherapy in the high GADD45B expression group was superior to the low GADD $45 B$ expression group. This result would provide stage II patients with a better selection for adjuvant chemotherapy to promote clinical outcomes and decrease overtreatment and cost. It was found that the stage II patients with low expression GAD45BB could appropriately change their chemotherapy regime or simply "look and wait." Moreover, if we want to translate the finding 
into a better treatment strategy, more cohorts and larger size cases are needed and a related prospective study is also necessary.

This study has some limitations. First, our validation cohorts only consist of stage II and liver metastatic CRC patients and lack the other stages. Therefore, the next step should be to complete the CRC cohort establishment of stage I, stage III, and even precancerous stages to further vindicate the GADD $45 B$ expression characteristics in the development of CRC. In addition, all the cases in the cohort were collected from one single institution, which means further validations form multicenter and larger sample size cohorts are needed.

Currently, checkpoint inhibitor treatment is a major problem in tumor immunotherapy. Although immunotherapy has not been carried out in CRC widespread, it was shown that GADD45B could modulate innate immune checkpoint functions, which are amenable to therapeutic intervention to reprogram tumor-associated macrophages and ultimately overcome tumor microenvironments dependent on immunosuppression $[17,21]$. Moreover, as mentioned in the discussion above, inhibition of the $G A D D 45 B$ expression leads to suppression of proliferation and further prohibits tumor progression. Collectively, GADD $45 B$ is not only a prognostic factor in CRC but also a promising potential treatment target in the future.

\section{Conclusions}

The results suggest that a high expression level of $G A D D 45 B$ is an independent prognostic factor of decreased OS and PFS in stage II CRC patients. The stage II CRC patients with high GADD45B expression may benefit from adjuvant chemotherapy.

Supplementary Materials: The following are available online at http:/ /www.mdpi.com/2073-4425/9/7/361/s1. Table S1: Cox analyses of potential prognostic factors for overall survival in the stage II CRC cohort, Table S2: Cox analyses of potential prognostic factors for progression-free survival in the stage II CRC cohort; Table S3: Colon cancer and rectal cancer samples provided by the TCGA project.

Author Contributions: Conceived and designed the experiments: Z.Z., Y.G., S.Z., X.W. Performed the experiments: Z.Z., X.G., X.L., R.Y. Analyzed the data: Z.Z., Z.L., Z.J., H.L., M.Y., C.L.

Funding: National Key Research and Development Program of the Ministry of Science and Technology of China (2016YFC0905303), CAMS Innovation Fund for Medical Sciences (CIFMS) (2016-I2M-1-001), and the Beijing Science and Technology Program (D17110002617004).

Acknowledgments: This study was supported by the National Key Research and Development Program of the Ministry of Science and Technology of China (2016YFC0905303), CAMS Innovation Fund for Medical Sciences (CIFMS) (2016-I2M-1-001), and the Beijing Science and Technology Program (D17110002617004). The authors would like to thank the donors and the Cancer Institute \& Hospital, Chinese Academy of Medical Sciences, Peking Union Medical College, Beijing, China for tumor specimens, which were provided for the study, and acknowledge the efforts of The Cancer Genomic Atlas (TCGA) project and the program development of the GEPIA by Zhang et al. The interpretation of the data is the sole responsibility of the authors.

Conflicts of Interest: The authors declare no conflict of interest.

\section{References}

1. Chen, W.; Zheng, R.; Baade, P.D.; Zhang, S.; Zeng, H.; Bray, F.; Jemal, A.; Yu, X.Q.; He, J. Cancer statistics in China, 2015. CA Cancer J. Clin. 2016, 66, 115-132. [CrossRef] [PubMed]

2. Weiser, M.R.; Landmann, R.G.; Kattan, M.W.; Gonen, M.; Shia, J.; Chou, J.; Paty, P.B.; Guillem, J.G.; Temple, L.K.; Schrag, D.; et al. Individualized prediction of colon cancer recurrence using a nomogram. J. Clin. Oncol. 2008, 26, 380-385. [CrossRef] [PubMed]

3. Quasar Collaborative, G.; Gray, R.; Barnwell, J.; McConkey, C.; Hills, R.K.; Williams, N.S.; Kerr, D.J. Adjuvant chemotherapy versus observation in patients with colorectal cancer: A randomised study. Lancet 2007, 370, 2020-2029. [CrossRef]

4. Rosmarin, D.; Palles, C.; Church, D.; Domingo, E.; Jones, A.; Johnstone, E.; Wang, H.; Love, S.; Julier, P.; Scudder, C.; et al. Genetic markers of toxicity from capecitabine and other fluorouracil-based regimens: Investigation in the QUASAR2 study, systematic review, and meta-analysis. J. Clin. Oncol. 2014, 32, 1031-1039. [CrossRef] [PubMed] 
5. Salvador, J.M.; Brown-Clay, J.D.; Fornace, A.J., Jr. Gadd45 in stress signaling, cell cycle control, and apoptosis. Adv. Exp. Med. Biol. 2013, 793, 1-19. [PubMed]

6. Liebermann, D.A.; Hoffman, B. Myeloid differentiation (MyD)/growth arrest DNA damage (GADD) genes in tumor suppression, immunity and inflammation. Leukemia 2002, 16, 527-541. [CrossRef] [PubMed]

7. Liebermann, D.A.; Hoffman, B. Myeloid differentiation (MyD) primary response genes in hematopoiesis. Blood Cells Mol. Dis. 2003, 31, 213-228. [CrossRef]

8. Ju, S.; Zhu, Y.; Liu, L.; Dai, S.; Li, C.; Chen, E.; He, Y.; Zhang, X.; Lu, B. GADD45B and Gadd45g are important for anti-tumor immune responses. Eur. J. Immunol. 2009, 39, 3010-3018. [CrossRef] [PubMed]

9. Inowa, T.; Hishikawa, K.; Matsuzaki, Y.; Isagawa, T.; Takeuchi, T.; Aburatani, H.; Kitamura, T.; Fujita, T. GADD45 $\beta$ determines chemoresistance and invasive growth of side population cells of human embryonic carcinoma. Stem Cells Int. 2010, 2010, 782967. [CrossRef] [PubMed]

10. Ou, D.L.; Shen, Y.C.; Yu, S.L.; Chen, K.F.; Yeh, P.Y.; Fan, H.H.; Feng, W.C.; Wang, C.T.; Lin, L.I.; Hsu, C.; et al. Induction of DNA damage-inducible gene GADD $45 \beta$ contributes to sorafenib-induced apoptosis in hepatocellular carcinoma cells. Cancer Res. 2010, 70, 9309-9318. [CrossRef] [PubMed]

11. Michaelis, K.A.; Knox, A.J.; Xu, M.; Kiseljak-Vassiliades, K.; Edwards, M.G.; Geraci, M.; Kleinschmidt-DeMasters, B.K.; Lillehei, K.O.; Wierman, M.E. Identification of growth arrest and DNA-damage-inducible gene $\beta$ (GADD45 $\beta$ ) as a novel tumor suppressor in pituitary gonadotrope tumors. Endocrinology 2011, 152, 3603-3613. [CrossRef] [PubMed]

12. Wang, L.; Xiao, X.; Li, D.; Chi, Y.; Wei, P.; Wang, Y.; Ni, S.; Tan, C.; Zhou, X.; Du, X. Abnormal expression of GADD45B in human colorectal carcinoma. J. Transl. Med. 2012, 10, 215. [CrossRef] [PubMed]

13. Tang, Z.; Li, C.; Kang, B.; Li, C.; Zhang, Z. GEPIA: A web server for cancer and normal gene expression profiling and interactive analyses. Nucleic Acids Res. 2017, 45, W98-W102. [CrossRef] [PubMed]

14. IHC Staining Protocol for Paraffin, Frozen and Free Floating Sections. Available online: https:/ /www.abcam. com/protocols/immunostaining-paraffin-frozen-free-floating-protocol (accessed on 18 July 2018).

15. Mylonas, I.; Schiessl, B.; Jeschke, U.; Vogl, J.; Makrigiannakis, A.; Kuhn, C.; Schulze, S.; Kainer, F.; Friese, K. Expression of inhibin/activin subunits $\alpha$ (-alpha), $\beta A$ (-betaA), and $\beta B(-\beta B)$ in placental tissue of normal, preeclamptic, and HELLP pregnancies. Endocr. Pathol. 2006, 17, 19-33. [CrossRef] [PubMed]

16. Burges, A.; Shabani, N.; Bruning, A.; Mylonas, I. Inhibin- $\beta$ A and $-\beta B$ subunits in normal and malignant glandular epithelium of uterine cervix and HeLa cervical cancer cell line. Arch. Gynecol. Obstet. 2011, 284, 981-988. [CrossRef] [PubMed]

17. Verzella, D.; Bennett, J.; Fischietti, M.; Thotakura, A.K.; Recordati, C.; Pasqualini, F.; Capece, D.; Vecchiotti, D.; D'Andrea, D.; Di Francesco, B.; et al. GADD45 $\beta$ loss ablates innate immunosuppression in cancer. Cancer Res. 2018, 78, 1275-1292. [CrossRef] [PubMed]

18. Lee, K.W.; Lee, S.S.; Hwang, J.E.; Jang, H.J.; Lee, H.S.; Oh, S.C.; Lee, S.H.; Sohn, B.H.; Kim, S.B.; Shim, J.J.; et al. Development and validation of a six-gene recurrence risk score assay for gastric cancer. Clin. Cancer Res. 2016, 22, 6228-6235. [CrossRef] [PubMed]

19. Li, L.; Cai, S.; Liu, S.; Feng, H.; Zhang, J. Bioinformatics analysis to screen the key prognostic genes in ovarian cancer. J. Ovarian Res. 2017, 10, 27. [CrossRef] [PubMed]

20. Kalluri, H.S.G.; Kuo, J.S.; Dempsey, R.J. Effect of D609 on the expression of GADD45 $\beta$ protein: Potential inhibitory role in the growth of glioblastoma cancer stem like cells. Eur. J. Pharmacol. 2016, 791, 510-517. [CrossRef] [PubMed]

21. Salerno, D.M.; Tront, J.S.; Hoffman, B.; Liebermann, D.A. Gadd45a and GADD45B modulate innate immune functions of granulocytes and macrophages by differential regulation of p38 and JNK signaling. J. Cell. Physiol. 2012, 227, 3613-3620. [CrossRef] [PubMed]

(C) 2018 by the authors. Licensee MDPI, Basel, Switzerland. This article is an open access article distributed under the terms and conditions of the Creative Commons Attribution (CC BY) license (http:// creativecommons.org/licenses/by/4.0/). 